\title{
Crack extension near an auxetic particle using symmetric Galerkin boundary elements
}

\author{
J. R. Berger ${ }^{1}$, M. Adam ${ }^{1}$, I. Reimanis ${ }^{1}$ \& A.-V. Phan ${ }^{2}$ \\ ${ }^{1}$ Colorado School of Mines, Golden, Colorado, USA \\ ${ }^{2}$ University of South Alabama, Mobile, Alabama, USA
}

\begin{abstract}
The effect of either a single inclusion or groups of inclusions on crack propagation has been studied effectively using symmetric Galerkin boundary elements (SGBEM) and modified quarter-point crack tip elements. Typical results show that an inclusion can decrease the crack-tip stress intensity as the crack approaches an inclusion, followed by deflection of the crack. Interestingly, as the crack extends beyond the inclusion there can also be an amplification of stress intensity. These previous results have shown the great influence the presence of an inclusion may have on crack extension behavior. Here, we examine the influence of an auxetic particle on crack growth behavior. An auxetic material is a material which exhibits a negative Poisson ratio, so they exhibit lateral expansion upon longitudinal tensile loading, and also undergo lateral contraction under longitudinal compression. Such materials can exist in cellular form, or along specific axes in certain crystals. The objective of the present study is understanding the behavior of crack path and predict the crack growth direction in materials reinforced with auxetic particles. We will show the dramatic difference in crack path as compared to particles with positive Poisson ratios by showing results for crack extension in identical specimen geometries reinforced with typical (positive Poisson ratio) particles and auxetic particles.
\end{abstract}

\section{Introduction}

Poisson's ratio is defined as the negative of the ratio of lateral and axial strains under axial deformation. Most common materials undergo a transverse contraction when stretched in one direction; the magnitude of this transverse deformation is governed by Poisson's ratio. Poisson's ratio has two theoretical limits for a linear 
elastic, isotropic material: $-1 \leq \nu \leq 0.5$. The lower limit, $\nu \rightarrow-1$, is required for the strain energy to be a positive definite function (see, for example, [1]) while the upper limit, $\nu \rightarrow 0.5$, represents the incompressible limit for the material. For anisotropic solids, no such limits exist as discussed in [2].

A material that exhibits a negative Poisson's ratio is called auxetic: the material expands laterally upon longitudinal tensile loading and contracts laterally under longitudinal compression. A recent review article by [3] summarizes much of the state of the art as to current understanding of both natural and man-made auxetic materials. Some examples are silicon polymorphs such as those discussed in [4], zeolites as discussed in [5], and silicates as studied in [6]. Additionally, auxetic behavior can be created in foams and similar materials by varying the geometric structure, see [7]. Auxetic behavior has also been observed to occur locally on the nanoscale in certain materials as discussed in [8].

The motivation for the present paper is to investigate problems involving crack extension near auxetic particles. Of particular interest are the conditions required, in terms of elastic constants, for a crack to either be deflected from or attracted to an auxetic particle. Knowledge of such conditions may suggest potential toughening strategies in brittle materials reinforced with auxetic particles. We will use a symmetric Galerkin boundary element method for our crack extension simulations. We first verify our simulations by performing crack extension studies near isotropic inclusions, and compare our results with results available in the literature for these problems (for example, in $[9,10]$ ). We then investigate crack extension when the particle is auxetic, and present results in terms of elastic modulus mismatch for attracting or deflecting the crack from the particle. Finally, we investigate the behavior of the mode-I and mode-II stress intensity factors as the crack approaches the particle.

\section{Crack extension near a particle}

The general problem of a crack approaching a particle (or inclusion) is shown in Figure 1.

With reference to the figure, we will use $E_{p}$ and $\nu_{p}$ for the Young's modulus and the Poisson's ratio for the particle, and $E_{m}$ and $\nu_{m}$ those for the matrix material. Both materials are considered isotropic, and we will be interested in the crack extension behavior for various ratios of the elastic moduli as $\nu_{p}$ becomes negative. We consider the particle to be circular, and perfectly bonded to the matrix material, so at the matrix-particle interface we have

$$
u_{r}^{p}=u_{r}^{m}, \quad u_{\theta}^{p}=u_{\theta}^{m} \quad \text { and } \quad \sigma_{r r}^{p}=\sigma_{r r}^{m}, \quad \sigma_{r \theta}^{p}=\sigma_{r \theta}^{m}
$$

where $u_{r}, u_{\theta}$ are displacement components and $\sigma_{r r}, \sigma_{r \theta}$ are stress components in polar coordinates, and the superscripts $p$ and $m$ indicate the particle and matrix, respectively.

The general problem of a crack interacting with a particle has been studied analytically both for isotropic materials (see, for example, [11-13]) and for anisotropic problems (see, for example, [14]). 


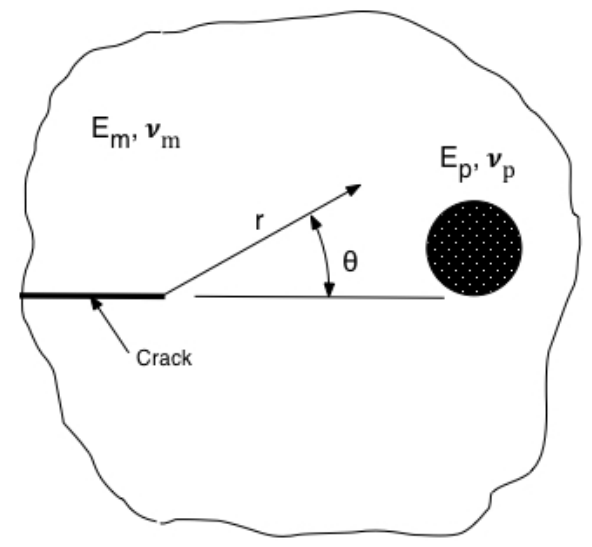

Figure 1: Crack approaching a particle.

The most relevant to the study reported here is the paper by [15] where the problem of a crack approaching either a coated or uncoated inclusion was studied. The authors found that the Poisson's ratio of the different phases (matrix and inclusion) could have a significant effect on the crack trajectory. For a crack approaching an uncoated elastic inclusion, the authors concluded that by simply modifying the Poisson ratio mismatch the rate at which the crack propagates, and the crack deflection/attraction mechanism can be controlled. We note that [15] only considered positive values for the Poisson ratio.

\section{Fracture analysis by the SGBEM}

The symmetric Galerkin formulation for boundary integral equations in twodimensional isotropic linear elastostatics has been extensively reported on in the literature and we will not review it here (see, for example, the book by [16]). The SGBEM has several advantages for crack-extension problems [10]: the formulation yields a symmetric coefficient matrix; no subdomains are required to solve fracture problems; no smoothness requirements are needed on the displacements for evaluating the hypersingular integrals; and a smoother solution is obtained near geometric discontinuities.

We will use three-noded quadratic elements for the boundary element calculations reported here. We approximate both the boundary and the boundary functions using this particular interpolation. Employing the parameter space $t \in$ $[0,1]$, and defining $t_{1}=0, t_{2}=1 / 2$ and $t_{3}=1$, the shape functions are defined as

$$
\psi_{1}(t)=(1-t)(1-2 t), \quad \psi_{2}(t)=4 t(1-t), \quad \psi_{3}(t)=t(2 t-1) .
$$


The approximate representations of the boundary and boundary functions are then given by

$$
\alpha_{i}=\sum_{\ell=1}^{3} \psi_{\ell}(t) \alpha_{i}^{\ell}
$$

where $\alpha_{i}$ can be the boundary displacement $u_{i}$, the boundary traction $t_{i}$, or the boundary geometry $x_{i}$, or $y_{i}$. $\alpha_{i}^{\ell}$ is the nodal value of $\alpha_{i}$.

Let the elastostatic problem be posed in the domain $\Omega$ with outer boundary $\Gamma=\partial \Omega$. Further, let the portion of the outer boundary with displacement boundary conditions be $\Gamma_{u}$ and the portion of the boundary with traction boundary conditions be $\Gamma_{t}$, so $\Gamma=\Gamma_{u} \cup \Gamma_{t}$. If a crack of boundary $\Gamma_{c}$ is added to the domain, the new total boundary becomes $\Gamma^{*}=\Gamma \cup \Gamma_{c}$. The crack is composed of two symmetrically loaded surfaces $\Gamma_{c}^{+}$and $\Gamma_{c}^{-}$which are initially coincident. Let $\Gamma_{t}^{*}=\Gamma_{t}+\Gamma_{c}^{+}$. In this case, the displacement and traction boundary integral equations (BIEs) are written as

$$
\begin{aligned}
\mathbf{U}_{k}^{*}(P) & =\mathbf{U}_{k}(P)+\int_{\Gamma_{c}^{+}} T_{k j}(P, Q) \Delta u_{j}(Q) \mathrm{d} Q=0 \\
\mathbf{T}_{k}^{*}(P) & =\mathbf{T}_{k}(P) \\
& +n_{\ell}^{+}(P) \int_{\Gamma_{c}^{+}} S_{k j \ell}(P, Q) \Delta u_{j}(Q) \mathrm{d} Q=0
\end{aligned}
$$

where $T_{k j}$ and $S_{k j l}$ are the usual Kelvin kernels used in boundary element analysis, $P, Q$ are the source and field point locations, respectively, and $n_{\ell}^{+}$is the outward normal vector to $\Gamma_{c}^{+}$. In these equations, the displacement jump vector $\Delta u_{j}$ across the crack surfaces is used as the unknown on the crack. As a result, only one crack surface, e.g., $\Gamma_{c}^{+}$, needs to be discretized. It is well known that the traction boundary integral equation, eq. (4), is essential for treating crack geometries.

The use of $\Delta u_{j}$ as the unknown on the crack as mentioned above is needed for obtaining a symmetric coefficient matrix. The symmetric-Galerkin formulation is given by

$$
\begin{gathered}
\int_{\Gamma_{u}} \psi_{k}(P) \mathbf{U}_{k}^{*}(P) \mathrm{d} P=0 \\
\int_{\Gamma_{t}^{*}} \psi_{k}(P) \mathbf{T}_{k}^{*}(P) \mathrm{d} P=0
\end{gathered}
$$

For standard fracture analysis problems, wherein the boundary condition on the crack is a specified traction, the symmetric-Galerkin procedure is remarkably simple: the above prescription (writing the traction equation on the crack surface) retains the symmetry, with the proviso that the unknowns on the fracture surface are now the jump in displacement, and the complementary variable is the sum of the known tractions. See for example, [17] and [18]. 
The important detail that requires discussion is the crack front treatment. As is well known, in linear elastic fracture mechanics the opening displacement at the crack front is non-analytic, behaving as $r^{1 / 2}$, where $r$ is the distance to the tip. Obtaining accurate stress intensity factors therefore requires that this behavior is incorporated into the numerical model. [19] and independently [20], provided an easy way to do this: they showed that by moving the mid-node coordinates $\left(x_{2}, y_{2}\right)$ three fourths of the way towards the tip, the parameter $t$ becomes $\sqrt{r / L}$, with $L$ the distance from $\left(x_{1}, y_{1}\right)$ to $\left(x_{3}, y_{3}\right)$. As a consequence, the leading order term in $\Delta u_{k}^{j}$ at $t=0$, which is $t$, is the correct square root of distance. Note however, that the next term, which is $t^{2}$, is $r / L$. Following [21] this term should vanish, and the modification presented below replaces this term with $(r / L)^{3 / 2}$.

For the new approximation, we keep the representation of $\Gamma(t)$ so that the property $t \approx \sqrt{r}$ remains, and the interpolation of the geometry remains quadratic. However for the crack opening displacement (COD, the difference in displacement on the two sides of the crack), we define new shape functions by adding a cubic term:

$$
\hat{\psi}_{2}(t)=-\frac{8}{3}\left(t^{3}-t\right), \quad \hat{\psi}_{3}(t)=\frac{4}{3}\left(4 t^{3}-t\right)
$$

This additional contribution accomplishes the cancelation of the $t^{2} \approx r$ term, without disturbing the interpolation, i.e., $\hat{\psi}_{\ell}\left(t_{m}\right)=\delta_{\ell m}$.

The results reported in this paper employ the Displacement Correlation Technique (DCT) to compute the mode-I and mode-II stress intensity factors, $K_{I}, K_{I I}$. With the modified quarter-point element, accurate results can be achieved, even with this very simple evaluation method. The general expression for the stress intensities by means of the DCT technique are

$$
\begin{aligned}
K_{I} & =\frac{\mu}{\kappa+1} \lim _{r \rightarrow 0} \sqrt{\frac{2 \pi}{r}} \Delta u_{n} \\
K_{I I} & =\frac{\mu}{\kappa+1} \lim _{r \rightarrow 0} \sqrt{\frac{2 \pi}{r}} \Delta u_{t}
\end{aligned}
$$

where $L$ is the crack length and $\Delta u_{n}$ and $\Delta u_{t}$ are the normal and tangential components of the displacement jump vector, respectively, $\kappa=3-4 \nu$ for plane strain and $\kappa=\frac{3-\nu}{1+\nu}$ for plane stress. As discussed in [22], with the modified quarter-point these become

$$
\begin{aligned}
K_{I} & =\frac{\mu}{3(\kappa+1)} \sqrt{\frac{2 \pi}{L}}\left(8 \Delta u_{n}(B)-\Delta u_{n}(C)\right. \\
K_{I I} & =\frac{\mu}{3(\kappa+1)} \sqrt{\frac{2 \pi}{L}}\left(8 \Delta u_{t}(B)-\Delta u_{t}(C)\right.
\end{aligned}
$$

where node $B$ is at the quarter-point and node $C$ is at the far end of the element, away from the crack tip. Thus, $K_{I}, K_{I I}$ are given directly in terms of the nodal values of the crack opening displacement on the crack tip element. 
In order to extend the crack, a crack extension criterion must be selected. Here, we use the maximum principal stress criterion of [23] where the crack grows in a direction perpendicular to the maximum principal stress. The crack extension angle, $\theta_{c}$ can be calculated from the condition $\sigma_{r \theta}=0$ ahead of the crack as

$$
K_{I} \sin \theta_{c}+K_{I I}\left(3 \cos \theta_{c}-1\right)=0
$$

The crack extension simulation can proceed in a straightforward manner by performing the SGBEM analysis, computing $K_{I}, K_{I I}$ from eq. (9), computing $\theta_{c}$ from eq. (10), then extending the crack by a small amount $\Delta a$ in the direction of the crack extension. The crack extension is accomplished by adding a new modified quarter point element to the tip of the crack, and reverting the previous cracktip element to a standard element. For the simulations reported on here, crack extension will only occur in the matrix material, we terminate our calculations if the crack impinges on an interface due to the increased complexity of the crack-tip stress field. As noted in [10], the SGBEM with modified quarter-point crack elements is capable of producing highly accurate results for both crack path and stress intensities, even when the crack tip is extremely close to interface boundaries.

\section{Effect of a single auxetic particle on the crack path}

In order to validate our analysis, we will use a problem geometry identical to that used in [10], see Figure 2, and benchmark our analysis against results for non-auxetic particles. With reference to the figure, we will use $L=0.150 \mathrm{~m}$ and

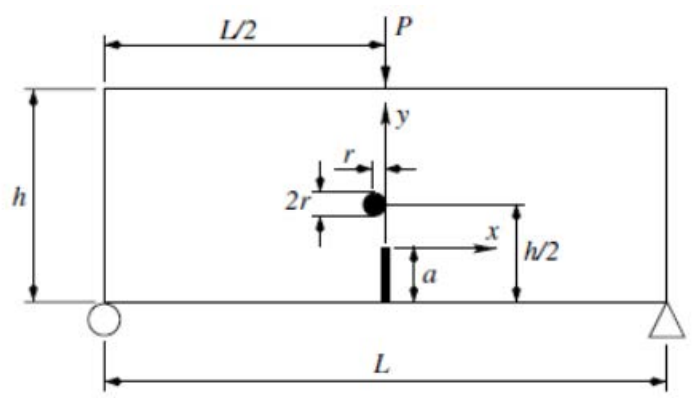

Figure 2: Three-point bend specimen.

$h=0.04 \mathrm{~m}$ in our calculations. The origin of coordinates is located at the crack tip, and the initial crack length is $h / 4$. The changes in crack length in the $x$ and $y$ directions are normalized with respect to the half-length $L / 2$ and the height of the beam, $h$, respectively. The particle radius is denoted by $r_{p}$ which is selected to be very small $\left(r_{p}=0.001 \mathrm{~m}\right)$. The particle is centered vertically in the beam but offset from the crack path an amount $x=-r_{p}$ from the $y$-axis. 
We first consider non-auxetic particles, with $\nu_{p} / \nu_{m}=1$, and compute the crack path when $E_{p} / E_{m}$ varies from 2 to 16 . We use 193 quadratic elements on the outer boundary for this problem, 64 quadratic elements for the particle, and initially 10 elements for the crack. We take crack extension increments of $\Delta a=0.03 \mathrm{~mm}$. These values provided good agreement with published results. The results for the crack path are shown in Figure 3, where the results from [10] are also plotted, but are directly on top of the results of our calculations. As such, we are confident in the accuracy of the results for the subsequent analyses reported here. Note in the

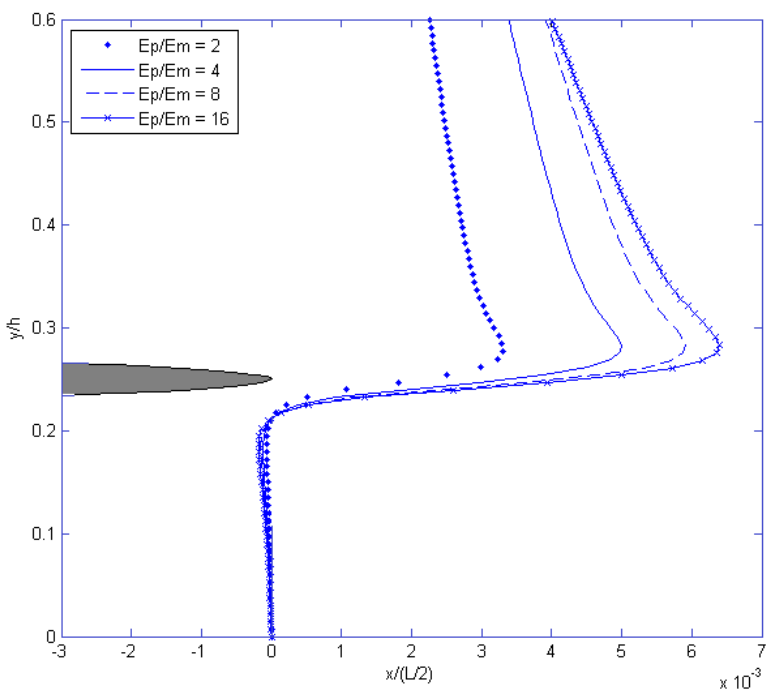

Figure 3: Comparison of results with [10] for non-auxetic particle-crack interaction, $\nu_{p} / \nu_{m}=1$. Note that the horizontal scale is expanded to emphasize the crack path.

figure that for $2 \leq E_{p} / E_{m} \leq 16$ the crack is always deflected away from the particle when $\nu_{p} / \nu_{m}=1$ with the amount of deflection increasing with $E_{p} / E_{m}$. It was also noted in [10] that the ratio $\nu_{p} / \nu_{m}$ only has a slight influence on crack deflection when $E_{p} / E_{m}$ is large, but has a pronounced effect on the crack path at lower values of $E_{p} / E_{m}$. This is consistent with the results reported in [15].

We next perform an analysis similar to our benchmark analysis, but the particle is taken to be auxetic: $\nu_{p} / \nu_{m}=-1$. We investigate the effects of the mismatch in Young's modulus on crack extension by again varying $E_{p} / E_{m}$ from 2 to 16 . Our simulation results are shown in Figure 4. In contrast to the results shown in Figure 3 for the non-auxetic particle, we see that for lower values of $E_{p} / E_{m}$ $\left(E_{p} / E_{m}=2,4\right)$ the auxetic particle actually attracts the crack. When the particle 
is stiffer than the matrix material $\left(E_{p} / E_{m}=8,16\right)$ the crack is deflected away from the particle as it is in the case of the non-auxetic particle.

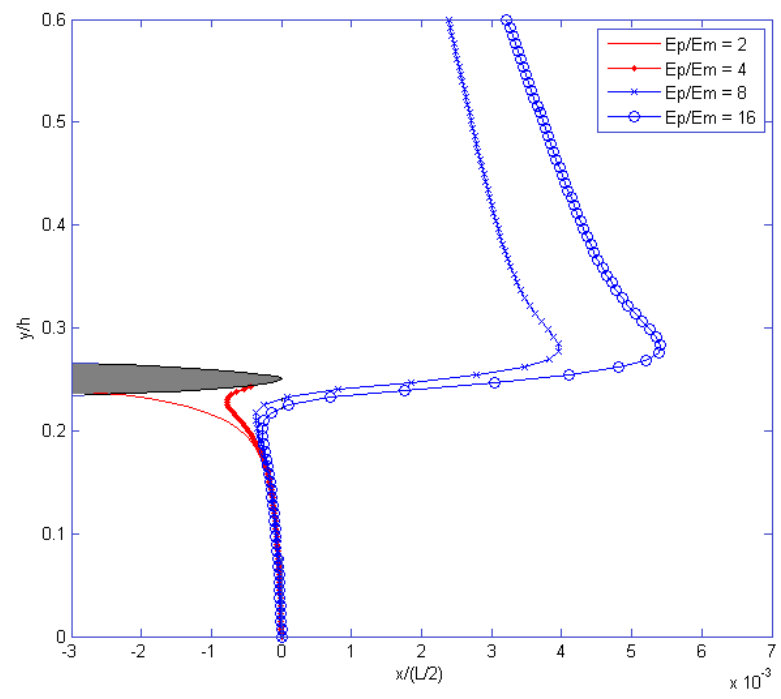

Figure 4: Crack extension near an auxetic particle, $\nu_{p} / \nu_{m}=-1$.Note that the horizontal scale is expanded to emphasize the crack path.

\section{Summary}

We employed a symmetric Galerkin based boundary element method to analyze crack extension near an auxetic particle. We found that the crack extension behavior can be dramatically different near an auxetic particle when compared to extension behavior near a non-auxetic particle. For values of $E_{p} / E_{m}=2,4$ we found that the crack was attracted to the particle when $\nu_{p} / \nu_{m}=-1$, yet when $\nu_{p} / \nu_{m}=1$ the crack is deflected away from the particle. We also found that when $E_{p} / E_{m}=8,16$ the crack was deflected away from the particle when $\nu_{p} / \nu_{m}= \pm 1$. This suggests strategies for pinning extending matrix cracks by employing auxetic particles that tend to attract the crack. Indeed, we found that a soft particle $\left(E_{p} / E_{m}=0.5\right)$ will attract the crack when $\nu_{p} / \nu_{m}= \pm 1$, but if the particle was hard $\left(E_{p} / E_{m}=0.5\right)$ the crack was only attracted when $\nu_{p} / \nu_{m}=-1$.

\section{Acknowledgement}

The authors acknowledge the support of the Army Research Office, grant number DAAD19-01-1-0590, to the Colorado School of Mines. 


\section{References}

[1] Fung, Y. C., Foundations of Solid Mechanics, Prentice Hall, Englewood Cliffs, NJ (1965).

[2] Ting, T. C. T. and Chen, T., Poisson's ratio for anisotropic elastic materials can have no bounds, Quarterly Journal of Mechanics and Applied Mathematics 58: 73-82 (2005).

[3] Yang, W., Li, Z.-M., Shi, W., Xie, B.-H. and Yang, M.-B., Review on auxetic materials, Journal of Materials Science 39: 3269-3279 (2004).

[4] Kimizuka, H., Kaburaki, H. and Kogure, Y., Mechanism for negative Poisson ratios over the $\alpha-\beta$ transition of cristobalite, $\mathrm{SiO} 2$ : A molecular-dynamics study, Physical Review Letters 84: 5548-5551 (2000).

[5] Grima, J. N., Jackson, R., Alderson, A. and Evans, K. E., Do zeolites have a negative Poisson's ratios?, Advanced Materials 12: 1912-1918 (2000).

[6] Grima, J. N., Gatt, R., Alderson, A. and Evans, K. E., On the origin of auxetic behavior in the silicate $\alpha$-cristobalite, Journal of Material Chemistry 15: 4003-4005 (2005).

[7] Grima, J. N. and Evans K. E., Auxetic behavior from rotating squares, Journal of Materials Science Letters 19: 1563-1565 (2000).

[8] Alderson, A., Alderson, K. L., Evans, K. E., Grima, J. N., Williams, M. R. and Davies, P. J., Modeling the deformation mechanisms, structure-property relationships and applications of auxetic nanomaterials, Phys. Stat. Sol. B 242: 499-508 (2005).

[9] Bush, M. B., The interaction between a crack and a particle cluster, International Journal of Fracture, 88: 215-232 (1997).

[10] Williams, R. C., Phan, A.-V., Tippur, H. V., Kaplan, T., and Gray, L. J., SGBEM analysis of crack-particle(s) interactions due to elastic constant mismatch, Engineering Fracture Mechanics 74: 314-331 (2007).

[11] Tamate, O., The effect of a circular inclusion on the stresses around a line crack in a sheet under tension, International Journal of Fracture Mechanics, 4: 257-266 (1968).

[12] Atkinson, C., The interaction between a crack and an inclusion, International Journal of Engineering Science, 10: 127-136 (1972).

[13] Erdogan, F., Gupta, G. D. and Ratwani, M., Interaction between a circular inclusion and an arbitrarily oriented crack, Journal of Applied Mechanics, 41: 1007-1013 (1974).

[14] Hwu, C., Liang, Y. K. and Yen, W. J., Interactions between inclusions and various types of cracks, International Journal of Fracture, 73: 301-323 (1995).

[15] Knight, M. G., Wrobel, L. C., Henshall, J. L., and De Lacerda, L. A., A study of the interaction between a propagating crack and an uncoated/coated elastic inclusion using the BE technique, International Journal of Fracture, 114: 47-61 (2002).

[16] Sutradhar, A., Paulino, G. H. and Gray, L. J., The Symmetric Galerkin Boundary Element Method, Springer Verlag, Berlin (2008). 
[17] Sirtori, S., Maier, G., Novati, G., and Miccoli, S., A Galerkin symmetric boundary element method in elasticity: formulation and implementation, International Journal for Numerical Methods in Engineering 35: 255-282 (1992).

[18] Bonnet, M., Maier, G., and Polizzotto, C., Symmetric Galerkin boundary element method, ASME Applied Mechanics Reviews 51: 669-704 (1998).

[19] Henshell, R. D. and Shaw, K. G., Crack tip finite elements are unnecessary, International Journal for Numerical Methods in Engineering, 9: 495-507 (1975).

[20] Barsoum, R. S., On the use of isoparametric finite elements in fracture mechanics, International Journal for Numerical Methods in Engineering, 10: 25-37 (1974).

[21] Gray, L.J. and Paulino, G.H., Crack tip interpolation, revisited, SIAM Journal on Applied Mathematics, 58: 428-455 (1998).

[22] Gray, L.J., Phan, A.-V., Paulino, G.H., and Kaplan, T., Improved quarterpoint crack tip element, Engineering Fracture Mechanics, 70: 269-283 (2003).

[23] Erdogan, F. and Sih, G. C., On the crack extension in plates under plane loading and transverse shear, Journal of Basic Engineering, 86: 519-527 (1963). 